\title{
$E 11853$
}

\section{Premixed edge-flames in spatially-varying straining flows*}

\author{
Jian-Bang Liu and Paul D. Ronney \\ Department of Aerospace and Mechanical Engineering \\ University of Southem California, Los Angeles, CA 90089-1453
}

\section{Introduction}

Flames subject to temporally and spatially uniform hydrodynamic strain are frequently used to model the local interactions of flame fronts with turbulent flow fields (Williams, 1985; Peters, 1986; Bradley, 1992). The applicability of laminar flamelet models in strongly turbulent flows have been questioned recently (Shay and Ronney, 1998) because in turbulent flows the strain rate $(\sigma)$ changes at rates comparable to $\sigma$ itself and the scale over which the flame front curvature and $\sigma$ changes is comparable to the curvature scale itself. Therefore quasi-static, local models of turbulent strain and curvature effects on laminar flamelets may not be accurate under conditions where the strain and curvature effects are most significant. The purpose of this study is to examine flames in spatially-varying strain and compare their properties to those of uniformly strained flames.

\section{Experimental approach}

As in previous work on nonpremixed edge flames (Shay and Ronney, 1998), premixed edge-flames were created using a counterflow slot-jet burner with the jet exits intentionally misaligned slightly to produce small strain rate gradients. Stable premixed edge-flames are anticipated because, according to theory (Vedarajan and Buckmaster, 1997; Vedarajan et al., 1998; Daou and Liñan, 1998a), the edge velocity is negative (retreating) in high-strain regions and positive (advancing) in low-strain regions, and thus the location where $\sigma(x)$ corresponds to zero edge velocity should be a stable equilibrium point. The global strain rate for counterflowing slot-jet streams of equal densities is given by $\sigma(x)=\left(V_{\text {upper }}+V_{\text {lower }}\right) / d(x)$, where $V_{\text {upper }}$ and $V_{\text {lowa }}$ are the upper and lower jet exit velocities and $d(x)$ is the jet spacing at location $x$.

The experimental apparatus consisted of two $7.6 \mathrm{~cm} \times 1.0 \mathrm{~cm}$ rectangular nozzles configured as a counterflow burner. Steel wool and aluminum honeycomb inside the nozzles ensured uniform exit flow. The nozzles were maintained at room temperature by water cooling. The lower nozzle was mounted on a rotation/translation stage with micrometers for adjusting the nozzle separation and wedge angle between the slot exits. Steel mesh screens were placed above and below the test section to minimize external disturbances and buoyancy effects. This apparatus was placed inside a steel box to isolate the flames from laboratory drafts and facilitate ventilation. Commercial mass flow controllers with accuracy $\pm 1 \%$ of full scale (verified by calibration with wet-test meters) delivered the combustible gases to the nozzles. The mass-flow controllers were commanded by a PC-based digital-to-analog converter board and custom software that enabled independent control of gas composition and $V$ for each nozzle. For twinflames, the two nozzles always had identical composition and $V$ to maintain symmetry. For single-flames, the upper nozzle contained the reactive flow and the lower non-combustible flow was the same inert gas ( $\mathrm{He}, \mathrm{N}_{2}$ or $\mathrm{CO}_{2}$ ) as used in the upper flow. In some single-flame cases, we employed $V_{\text {uppes }}>V_{\text {lower }}$ to move the flame farther from the upper nozzle and reduce heat losses (see Results).

\footnotetext{
'Spring Technical Meeting, joint U. S. Sections, Combustion Institute, Washington, D.C., March 15-17, 1999. Work supported by NASA-Lewis under grant NAG3-1523.
} 


\section{Results}

Edge-flames were observed for both single- and twin-flame configurations for all mixture families studied. For the single-flame configuration, the edge-flames exhibit a hook-like structure (Fig. 1, upper) with the tip bending downward toward the inert flow. This bending is expected since as $\sigma(x)$ increases, the burning velocity $\left(S_{L}\right)$ decreases and the equilibrium location of the flame (where $S_{L}$ equals the local flow velocity) is pushed toward the stagnation plane where the local velocity is lower. This hook-like structure is also predicted theoretically (Vedarajan and Buckmaster, 1997). For the twin premixed flame configuration, the two mirrorimage flame hooks join together at a corner-like edge (Fig. 1, middle and lower).

Sharp curvature such as that at the corner of twin edge-flames increases (decreases) the local chemical reaction rate relative to a flat flame in mixtures with Le $<1$ (Le $>1$ ) (Williams, 1985). While the classical models of curvature effects do not strictly apply to the corners of twin edge-flames because the flow at the comer is parallel to the flame front, nonetheless reaction is visibly strengthened for $\mathrm{CH}_{4} / \mathrm{O}_{2} / \mathrm{CO}_{2}$ mixtures ( $\left.\mathrm{Le}=0.6\right)$ (Fig. 2, middle) and weakened to the point of having an open edge in $\mathrm{C}_{3} \mathrm{H}_{8} / \mathrm{O}_{2} / \mathrm{He}$ mixtures (Le $\approx 3.0$ ) (Fig. 2, lower). These observations are also consistent with the well-known characteristic of strained twin premixed flames that for Le $<1$, the flames will merge before extinguishing, whereas for Le $>1$ extinguishment occurs before merging.

Figures 5a - $d$ show comparisons of $\sigma_{\text {ext }}$ for uniformly strained twin flames and $\sigma_{\text {edge }}$ for twin edge-flames. For practically all cases $\sigma_{\text {ext }}$ is lower for the edge-flame, with the difference being much greater for higher Le. For $\mathrm{CH}_{4}$-air mixtures $(\mathrm{Le} \approx 0.9)$, the difference does not seem to be nearly a factor of two as predicted for Le = 1 (Vedarajan et al., 1998; Daou and Liñan, 1998a). Also, $\sigma_{\text {ex }} / \sigma_{\text {odpe }}$ is close to unity for $L e=0.6$, whereas Daou and Liñán predict $\sigma_{\text {ext }} / \sigma_{\text {edpe }}$ 0.7 for this case. Otherwise, the results are very consistent with experiments in that $\sigma_{\text {ex }} j \sigma_{\text {edge }}$ decreases with increasing Le and reaches a nearly constant value of about 0.5 for sufficiently high Le. Figures 5a-d show that there is no significant dependence of $\sigma_{e x t}$ on the wedge angle between the nozzles, and thus on the strain rate gradient. The same conclusion was reached in our non-premixed edge-flame study (Shay and Ronney, 1998). There is no gradual transition from edge-flame to uniform-strain behavior as the divergence angle decreases. Thus, edgeflames are distinct from uniformly-strained flames; each type of flame exhibits consistent but different response to strain.

For the single flame configuration (Figs. $6 a-d), \sigma_{\text {ext }}$ is practically the same for edgeflames and uniformly-strained flames, with the edge-flame probably slightly weaker. This is consistent with Vedarajan and Buckmaster (1997), who predicted $\sigma_{\text {ext }}$ is only $7 \%$ lower for single edge-flames in Le $=1$ mixtures. Again, the wedge angle has almost no effect on these results and in this case Le has no significant effect either. Unfortunately, no predictions for single edgeflames with $L e \neq 1$ are available for comparison.

\section{Discussion and conclusions}

Experiments were conducted to observe the effect of strain rate gradients on steady strained premixed flames. Edge-flames were observed for both single and twin premixed-flame configurations. Hook-like structures were observed for the single-flame configuration and corner-like structures were observed for twin-flames. Video images and interferograms indicated a sharp transition from non-burning to burning regions at the flame edge.

For the twin-flame configuration, the strain rate at the edge of stationary edge-flames $\left(\sigma_{\text {coge }}\right)$ was nearly always lower than the extinction strain rates of uniformly strained flames in the same mixture $\left(\sigma_{\text {oxt }}\right)$, though theory (Vedarajan et al., 1998; Daou and Liñán, 1998a) predicts a factor of about two difference for $\mathrm{Le}=1$ whereas experimentally a difference of this magnitude was observed only at somewhat higher $L$ e. For the single-flame configuration $\sigma_{\text {edec }}$ was only very slightly lower than $\sigma_{\text {ext }}$, which is also consistent with the theoretical predictions for Le $=1$ (Vedarajan and Buckmaster, 1997). In this case, Le had practically no influence on the comparison. For both cases the strain rate gradient had no significant effect on $\sigma_{\text {edge }}$ and there 
was no gradual transition from edge-flame to uniformly strained flame behavior as the wedge angle was decreased, indicating that premixed edge-flames are distinctly different flame structures from uniformly strained flames.

The extinction strain rates of edge-flames and uniform flames are similar in many cases, thus the response of turbulent flames might be modeled reasonably well using properties measured for uniformly-strained flames. Consequently, laminar flamelet models of premixed turbulent combustion may be approximately valid up to conditions near the local quenching condition (except for mixtures with particularly low Le, e.g., lean $\mathrm{H}_{2}$-air, or particularly high Le, e.g., lean gasoline-air), a position advocated in a recent review (Bradley, 1992), when the global strain rate is properly characterized. Of course, this conclusion might not apply in large strain rate gradients or in combined spatially- and temporally-varying flows. It is interesting that a different conclusion was reached for non-premixed flames (Shay and Ronney, 1998) because the differences between edge-flames and uniformly-strained flames was greater in that case and was observed for all Le. This is consistent with a recent numerical study of nonpremixed edgeflames by Daou and Liñan (1998b), who predicted that for fuel Lewis numbers of 3/8, 1 and $13 / 8$, the corresponding values of $\sigma_{\text {edge }} / \sigma_{\text {ext }}$ were $0.52,0.41$ and 0.28 , respectively. Thus a comparison of Daou and Liñan's work for nonpremixed (1998b) and premixed (1998a) edgeflames shows that the distinction between edge-flames and uniform flames in terms of $\sigma_{\text {edsof }} / \sigma_{\text {ext }}$ is greater for nonpremixed flames and extends across all Le, which is entirely consistent with our experimental observations for premixed flames (this work) and nonpremixed flames (Shay and Ronney, 1998).

\section{References}

Bradley, D. (1992). How fast can we burn? Twenty-Fourth Symposium (International) on Combustion, Combustion Institute, Pittsburgh, p. 247.

Daou, J. and Liñan, A. (1998a). Ignition and extinction fronts in counterflowing premixed reactive gases. Manuscript in review.

Daou, J. and Liñán, A. (1998b). The role of unequal diffusivities in ignition and extinction fronts in strained mixing layers. Manuscript in review.

Peters, N. (1986). Laminar flamelet concepts in turbulent combustion. Twenty-first Symposium (International) on Combustion, Combustion Institute, Pittsburgh, p. 1231.

Shay, M. L. and Ronney, P. D. (1998). Nonpremixed flames in spatially-varying straining flows. Combustion and Flame 112, 171.

Vedarajan, T. G. and Buckmaster, J. D. (1998). Edge-flames in homogeneous mixtures. Combust. Flame 114, 267.

Vedarajan, T. G., Buckmaster, J. D. and Ronney, P. D. (1998). "Two-dimensional Failure Waves and Ignition Fronts in Premixed Combustion," Twenty-Seventh Symposium (International) on Combustion, to appear.

Williams, F. A. (1985). Combustion Theory, 2nd ed., Benjamin-Cummins. 